
Polymer Films 
This page intentionally left blank 


\section{Plasma \\ Polymer Films}

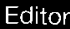

Hynek Biederman Charles University, Czech Republic

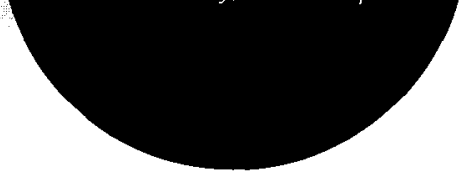

Imperial College Press 


\section{Published by}

Imperial College Press

57 Shelton Street

Covent Garden

London WC2H 9HE

\section{Distributed by}

World Scientific Publishing Co. Pte. Ltd.

5 Toh Tuck Link, Singapore 596224

USA office: Suite 202, 1060 Main Street, River Edge, NJ 07661

UK office: 57 Shelton Street, Covent Garden, London WC2H 9HE

\section{British Library Cataloguing-in-Publication Data}

A catalogue record for this book is available from the British Library.

\section{PLASMA POLYMER FILMS}

Copyright $\odot 2004$ by Imperial College Press

All rights reserved. This book, or parts thereof, may not be reproduced in any form or by any means, electronic or mechanical, including photocopying, recording or any information storage and retrieval system now known or to be invented, without written permission from the Publisher.

For photocopying of material in this volume, please pay a copying fee through the Copyright Clearance Center, Inc., 222 Rosewood Drive, Danvers, MA 01923, USA. In this case permission to photocopy is not required from the publisher.

ISBN $1-86094-467-1$ 


\section{CONTENTS}

PREFACE

CHAPTER 1

INTRODUCTION

Hynek Biederman

\subsection{History}

1.2. What is a Plasma Polymer? 14

1.3. Deposition Systems for Plasma Polymerization 16

1.4. Deposition (Plasma) Process Parameters 20

1.5. Models of Plasma Polymerization 20

1.6. Types of Plasma Polymers and their Characterization 21

1.7. Conclusions 22

$\begin{array}{ll}\text { References } & 22\end{array}$

CHAPTER 2

PLASMA DEPOSITION OF FLUOROPOLYMER

FILMS IN DIFFERENT GLOW DISCHARGE REGIMES

Pietro Favia

2.1. Introduction 25

2.2. On the Jargon of PE-CVD Processes 26

2.3. Continuous, Afterglow and Modulated PE-CVD 27

2.3.1. Fluorocarbon Monomers 28

2.4. Diagnostic Techniques for PE-CVD Processes of Fluoropolymers 29

2.4.1. Surface and Bulk Analysis $\quad 30$

2.4.2. Plasma Diagnostic Techniques 31

2.5. Active Species in Fluorocarbon Plasmas 33

2.5.1. Additives to Drive the $F / C F_{x}$ Density Ratio of a Fluorocarbon Discharge 34

2.6. Relevant Parameters in PE-CVD Processes of Fluoropolymers 37

2.6.1. Ion Bombardment and Substrate Temperature 37

2.6.2. Power, Flow Rate, Pressure and Combined Parameters 39

2.6.3. Distance of the Substrate from the Glow 40

2.6.4. Duty Cycle and Period in Modulated Plasmas 42

2.7. The Activated Growth Model 46 
2.8. Applications of Plasma-Deposited Fluoropolymers

2.8.1. Wettability of Fluorocarbon Coatings and Applications for Textiles and Paper

2.8.2. Biomedical Applications Proposed for Plasma-Deposited Fluoropolymers

2.9. Conclusive Remarks

References

CHAPTER 3

DEPOSITION OF SILICON CONTAINING FILMS AND FTIR DIAGNOSTICS

Yvan Segui and Patrice Raynaud

3.1. Introduction

$\begin{array}{ll}\text { 3.2. Precursors } & 58\end{array}$

3.3. Species Detected in Organosilicon Plasmas 62

3.3.1. Optical Emission Spectroscopy (OES) 62

3.3.2. Mass Spectroscopy 64

3.3.3. Infrared Absorbtion Spectroscopy of the Plasma 69

3.3.3.1. Principles of FTIR Diagnostic of the Plasma Phase 69

3.3.3.2. The Absorption Spectra $\quad 70$

3.3.3.3. Quantitative Analysis (Absorption Spectrometry) 71

3.3.3.4. Infrared Diagnostics of Organosilicon Plasma 72

3.4. Open Questions and Conclusions $\quad 76$

3.4.1. Species in the Gas Phase 76

3.4.2. Interface Plasma-Surface; Growing Mechanisms 77

$\begin{array}{ll}\text { References } & 79\end{array}$

CHAPTER 4

CORPUSCULAR DIAGNOSTICS OF PLASMA

POLYMERIZATION PROCESSES

Michael Zeuner

4.1. Corpuscular Diagnostics in Plasma Processing 85

4.2. Basic Processes in Particle Interaction 86

4.2.1. Gas Discharge Phenomena 87

4.2.2. Plasma Sheath Effects $\quad 89$

4.2.3. Surface Interaction $\quad 92$ 
4.3. Experimental Equipment for Corpuscular Diagnostics 95

4.3.1. Common Particle Collection by Film Deposition 96

4.3.2. Mass Spectrometrical Methods $\quad 99$

4.3.3. Energy Filter Techniques 105

4.4. Neutral Species Detection 109

4.4.1. Neutral Flux Detection and Partial Pressure Analysis 109

4.4.2. Threshold Ionisation Mass Spectrometry 113

4.4.3. Electron Attachment Mass Spectrometry 116

4.5. Process Ion Detection 117

4.5.1. RF Plasma Processing 118

4.5.2. RF Sputter Deposition $\quad 124$

4.5.3. Microwave Plasma Processing 127

4.5.4. Negative Ion Detection 129

4.5.5. Pulsed Plasma Operation 131

4.5.6. Plasma Ion Mass Spectra $\quad 135$

References $\quad 137$

CHAPTER 5

ELECTRICAL AND OPTICAL PROPERTIES OF PLASMA

POLYMERS 143

Jacek Tyczkowski

5.1. Introduction 143

5.2. Electronic Structure 144

5.2.1. Theoretical Models 144

5.2.2. Experimental Methods 148

5.2.2.1. Optical Spectra 148

5.2.2.2. Internal Photoemission $\quad 151$

5.2.2.3. Photoelectron Spectroscopies 154

5.2.2.4. Electrical Transport Methods 158

5.3. Amorphous Semiconductors 162

5.3.1. Band Model 162

5.3.2. Doping 164

$\begin{array}{ll}\text { 5.3.3. Alloying } & 167\end{array}$

5.3.4. Charge Carrier Transport 170

$\begin{array}{ll}\text { 5.4. Amorphous insulators } & 174\end{array}$

5.4.1. Band Model 174

$\begin{array}{ll}\text { 5.4.2. Electrical Conductivity } & 177\end{array}$

5.4.2.1. Charge Carriers 178

5.4.2.2. Generation Mechanisms 179

5.4.2.3. Transport Mechanisms 182 
5.5. Amorphous Insulator - Amorphous Semiconductor Transition

5.6. Electronic Properties and Potential Applications

5.6.1. Electrical Properties

5.6.1.1. Low Conducting Films

5.6.1.2. High Conducting Films

5.6.1.3. Intermediately Conducting Films

5.6.2. Optical and Optoelectronic Properties

5.6.2.1. Coatings and Films for Optical Devices 196

5.6.2.2. Integrated Optics

5.6.2.3. Optoelectronic Devices

5.6.3. Current Problems

References

CHAPTER 6

PULSED PLASMA POLYMERIZATIONS

Richard B. Timmons and Andrew J. Griggs

6.1. Introduction

6.1.1. Controlling Film Chemistry during Plasma Polymerizations

6.1.2. The Pulsed Plasma Approach

6.2. Experimental Considerations

6.2.1. Power Input Considerations

6.2.2. Film Formation Rates

6.2.3. Mean Roughness of Polymer Films Deposited under Pulsed Operation

6.2.4. Film Adhesion and Film Stability

6.3. Results

6.3.1. Some Examples of Film Chemistry Control under Pulsed Plasma Conditions

6.3.2. Film Formation Rates

6.3.3. Polymerization under Ultra Low Power Input Conditions

6.3.4. Film Composition Obtained under Equivalent Power

Input Conditions during Pulsed and CW Plasma

Polymerizations

6.4. Applications

6.4.1. Biomaterials

6.4.2. Thin Layer Adhesives

6.4.3. Synthesis of Super Hydrophobic Surfaces

6.4.4. Low Dielectric Constant Films

6.4.5. Electrically Conductive Polymers 
6.5. Discussion

References

CHAPTER 7

DEGRADATION AND STABILITY OF PLASMA POLYMERS

Andreas Holländer and Jörg Thome

7.1. Introduction

7.2. Mechanisms of Polymer Degradation 248

$\begin{array}{ll}\text { 7.2.1. General Considerations } & 249\end{array}$

7.2.2. Thermal Degradation 249

7.2.2.1. Hydrocarbon Polymer 250

7.2.2.2. Oxygen Containing Polymers 252

7.2.2.3. Aromatic Structures 255

7.2.2.4. Fluoropolymers 255

7.2.2.5. Silicone Polymers 256

$\begin{array}{ll}\text { 7.2.3. Oxidation } & 256\end{array}$

7.2.4. Photooxidation 259

7.3. The Degradation of Plasma Polymers 260

7.3.1. Structural Characteristics of Plasma Polymers 260

7.3.1.1. General Considerations 260

$\begin{array}{ll}\text { 7.3.1.2. Plasma Polymer Coatings } & 262\end{array}$

7.3.2. Aging of Plasma Polymers in Air 263

7.3.2.1. Hydrocarbon Plasma Polymers 263

7.3.2.2. Oxygen Containing Plasma Polymers 264

7.3.2.3. Nitrogen Containing Plasma Polymers 265

7.3.2.4. Fluoroaromatic Plasma Polymers 266

7.3.2.5. Siloxane and Silazane Plasma Polymers 266

7.3.2.6. Thermal Degradation of Plasma Polymers 267

7.3.2.7. Photooxidation of Plasma Polymers 268

7.3.3. General Features of Plasma Polymer Degradation 269

$\begin{array}{ll}\text { 7.4. Preparation of Stable Plasma Polymers } & 270\end{array}$

7.4.1. Reduction of the Radical Concentration $\quad 270$

7.4.1.1. Copolymerization 270

7.4.1.2. Thermal Annealing 270

7.4.1.3. Quenching of Trapped Radicals 271

7.4.2. Design Rules for Stable Plasma Polymers 271

7.5. Conclusions $\quad 272$

$\begin{array}{ll}\text { References } & 273\end{array}$ 
CHAPTER 8

APPLICATION OF ATMOSPHERIC PRESSURE DISCHARGE FOR PLASMA POLYMER PROCESSES

Masuhiro Kogoma

8.1. Introduction

8.3. Results and Discussion

8.3.1. FTIR and Emission Spectra

8.3.2. Mass Spectrometry of $\mathrm{C}_{2} \mathrm{H}_{4}$ and $\mathrm{C}_{2} F_{4}$ in He APG Discharge

8.3.3. TEOS deposition by APG Plasma

References

\section{CHAPTER 9}

HARD PLASMA POLYMERS, COMPOSITES AND PLASMA

POLYMER FILMS PREPARED BY RF SPUTTERING OF

CONVENTIONAL POLYMERS

Hynek Biederman, Pavel Kudrna and Danka Slavínská

9.1. Introduction

9.2. Hard Plasma Polymer and Hard Carbon Films 290

9.3. Composite Metal/Plasma Polymer Films 294

9.3.1. Deposition Techniques

9.3.1.1. Simultaneous Plasma Polymerization and Sputter-etching using an rf Discharge

9.3.1.2. Simultaneous Plasma Polymerization of an Organic Gas and Evaporation of a Metal

9.3.1.3. Plasma Polymerization of Metal Organic Compounds

9.3.1.4. Co-sputtering from Composite Metal/Polymer Target

9.3.1.5. De planar Magnetron Sputtering and Simultaneous Plasma Polymerization

9.3.2. Structure and Morphology of the Composite Films

9.3.3. Deposition Process and Composite Film Growth

9.3.4. Basic Physical Properties - Characterization

9.3.4.1. Structure, Morphology and Composition

9.3.4.2. Optical Properties

9.3.4.3. Electrical Properties

9.3.4.4. Mechanical Properties

9.3.4.5. Aging 
9.4. Plasma Polymer Films Prepared by rf Sputtering 309 9.4.1. Fluorocarbon Plasma Polymers $\quad 310$

9.4.2. Hydrocarbon Plasma Polymers 314

$\begin{array}{ll}\text { 9.5. Conclusions } & 317\end{array}$

Acknowledgements $\quad 317$

$\begin{array}{ll}\text { References } & 317\end{array}$

CHAPTER 10

BIOMEDICAL APPLICATIONS OF PLASMA-DEPOSITED

THIN FILMS

325

Kathryn J. Kitching, Vicki Pan and Buddy D. Ratner

10.1. Introduction 325

10.2. Biocompatibility: Relationship to Plasma Deposited Films 326

10.3. Applications for Cell and Tissue Culture 328

10.4. Applications for Controlled Release 334

10.5. Applications for Biosensors and Electrodes 337

10.6. Applications for Implanted and Blood-Contacting
Biomaterials

10.7. Applications for Contact Lenses 350

10.8. Non-fouling Coatings 357

10.9. Plasma Surface Treatments For Biorecognition 361

10.10. Patterned Plasma-Deposited Films 363

10.11. Perspectives and Conclusions 366

Acknowledgements $\quad 367$

$\begin{array}{ll}\text { References } & 367\end{array}$

$\begin{array}{ll}\text { INDEX } & 379\end{array}$ 
This page intentionally left blank 


\section{PREFACE}

The area of plasma polymerization is now well recognized as an important part of material science. Plasma polymerization spans over several fields of science, namely chemistry, physics and extends in recent years to biology. This book is intended not for beginners but for advanced students, $\mathrm{PhD}$ students and researchers from both academia and the industry who are already more or less in the field. The authors of the book try to give the readers the present state of the art and future trends of plasma polymerization.

However, in the first chapter Introduction, the accidental reader, who never came across this field before, may obtain very basic information and is directed to more detailed and basic books and review papers. The definition of the term plasma polymer is given, deposition systems, process parameters and models of plasma polymerization are described.

The second chapter Plasma deposition of fluoropolymer films in different glow discharge regimes focuses on continuous, afterglow and modulated (pulsed) discharges and discusses active species in fluorocarbon plasmas. Diagnostics techniques, namely OES (Optical emission spectroscopy) and AOES (Actinometric optical emission spectroscopy) are described. Active species in fluorocarbon plasmas are presented and process parameters mentioned. The activated growth model is introduced and the applications of fluoropolymers briefly reviewed.

In the third chapter Deposition of silicon containing films and FTIR diagnostics (Deposition from organosilicon/oxygen mixtures), most of the attention is devoted to the $\mathrm{SiO}_{\mathrm{x}} \mathrm{H}_{\mathrm{y}} \mathrm{C}_{\mathrm{z}}$ ( $\mathrm{z}, \mathrm{y}$ are almost zero) film deposition processes: the nature of the most frequently used precursors and their physical characteristics; type of plasma generation and the description of certain reactors. However, a summary of the literature on $\mathrm{SiN}_{x} \mathrm{H}_{y} \mathrm{C}_{z}$ is presented. The second part deals with the characterization of organosilicon - oxygenated gas mixture created plasmas. The principal species detected by the optical emission spectrometry, the mass spectrometry and the infrared absorption spectrometry (FTIR) are introduced. The application of FTIR in situ diagnostics is explained in more detail using the above examples.

In the fourth chapter Corpuscular diagnostics of plasma polymerization processes, experimental equipment for corpuscular diagnostics is described. Detection of neutral species and ions during the process are discussed in great detail. Examples are presented in terms of the discussion of particle fluxes to the growing film and the influence on the film morphology. 
In the fifth chapter Electrical and optical properties of plasma polymers, at first, the electronic structure of plasma polymers, investigated by various methods, such as electronic spectroscopies, photoinjection, optical absorption, etc, is described. The idea of two types of plasma polymers, namely amorphous semiconductors and amorphous insulators is discussed. For general view both inorganic films (as a-Si:H) and organic films (deposited from organic precursors) are presented. The electric conductivity, its mechanisms, as well as carrier generation and transport processes are further discussed. Attention is also paid to practical applications of the films in electronics such as field-effect transistors and amorphous "superlattice" structures. Basic dielectric parameters of the films and their dependence on deposition conditions, post-treatment of deposited films, aging processes, and environment influences (e.g. humidity) are also mentioned. At the end optical properties of plasma polymers are briefly summarized.

The sixth chapter Pulsed plasma polymerizations describes fundamental aspects of modulated plasmas, discusses potential advantages and limitations of this approach, and provides a thorough review of work to date in this area.

One of the most important features of the pulsed plasma approach is the convenient and high level of film chemistry controllability it provides during polymer formation. The inherent compositional controllability afforded by this approach is illustrated in this chapter with data from several laboratories involving a variety of monomers. This chapter also identifies and discusses differences in several important plasma variables when contrasting CW and pulsed plasma polymerizations and the potential influences on these variables on the respective film formation processes. The chapter concludes with a brief review of current and future applications which take particular advantage of the molecular surface tailoring possibilities provided by the pulsed plasma surface modification technique.

In the seventh chapter Stability of plasma polymers and plasma polymer coatings, thermal stability of plasma polymers (also versus the stability of conventional polymers) is discussed. Chemical (including photochemical) stability of plasma polymers is presented. Mechanism of physical and chemical processes during storage and use (long term stability, environmental stability) is discussed in detail. Stability of coating systems (layer adhesion, interphase adhesion) is described in detail.

In the eighth chapter Application of atmospheric discharge for plasma polymer processes, the basics of the atmospheric pressure glow plasma (APG) are presented. The studies of the deposition process of mainly organic (plasma 
polymer) films using emission spectrometry, IR absorption spectra and molecular beam (MB) mass spectrometry are described. The deposition system with parallel plate electrodes covered by glass insulator is described. Along with helium as carrier gas $\mathrm{C}_{2} \mathrm{H}_{4}$ and $\mathrm{C}_{2} \mathrm{~F}_{4}$, TEOS and some other monomers were used. Deposition processes for the first two monomers are discussed in detail.

The ninth chapter Hard plasma polymers, composites and plasma polymer films prepared by rf sputtering of conventional polymers pays attention at first to hydrocarbon plasma polymers and to the effect of energetic positive ion bombardment on the film hardness. Fluorocarbon plasma polymers are briefly mentioned. The basics of composite metal/plasma polymer films will be concisely presented: modes of their preparation, structure and morphology, anomalous electrical and optical properties. Composites: metal/hard plasma polymer and metal/hard carbon are described and their temperature and time stability compared to usual metal/plasma polymer composites.

Sputtering of polytetrafluoroethylene (PTFE) in argon and various gases as well as in self-sputtering mode is described. Composition of species in plasma volume and the growth of the fluorocarbon film in dependence on deposition parameters is discussed. The morphology (SEM), structure (FTIR, ESCA) and the electrical properties of the fluorocarbon plasma polymer films are described in detail. The main results in rf sputtering of polyethylene and some other polymers are briefly described.

The tenth chapter Biomedical applications of plasma-deposited thin films discusses the applications. The requirements for biocompatibility and how plasma deposited films meet those requirements are introduced. Following applications are described: Applications for cell culture, for controlled release of drugs, for biosensors, for implanted biomaterials and for contact lenses. Biorecognition and plasma-deposited films as well as patterned plasma deposited films are considered. In conclusion the perspectives are discussed.

The editor wishes to thank all his colleagues - and the co-authors of the book for their effort. Actually, the idea and need for such a book have been stimulated to a great extent by the Action COST 527: Plasma Polymers and Related Materials. The editor is further indebted to Dr. Pavel Kudrna for formatting the manuscript and to Eric Fuocco and Andrei Choukourov for reading and correcting it. The editor also thanks Dr. Christian Oehr for valuable discussions and comments related to the Chapter 1 - Introduction.

\section{CHARLES UNIVERSITY}

Prague, $31^{\text {st } J u l y, ~} 2002$

Hynek Biederman 